\title{
Article \\ Optofluidic Platform Based on Liquid Crystals in X-Cut Lithium Niobate: Thresholdless All-Optical Response
}

\author{
Fabrizio Ciciulla ${ }^{1}\left(\mathbb{D}\right.$, Annamaria Zaltron ${ }^{2}{ }^{\oplus}$, Riccardo Zamboni ${ }^{2,3}$, Cinzia Sada ${ }^{2}$, Francesco Simoni ${ }^{1,4, *}$, \\ Victor Yu. Reshetnyak ${ }^{5}$ and Liana Lucchetti ${ }^{1}$ \\ 1 Dipartimento SIMAU, Università Politecnica delle Marche, 60131 Ancona, Italy; \\ f.ciciulla@pm.univpm.it (F.C.); 1.lucchetti@staff.univpm.it (L.L.) \\ 2 Physics and Astronomy Department, University of Padova, 35131 Padova, Italy; \\ annamaria.zaltron@unipd.it (A.Z.); riccardo.zamboni@uni-muenster.de (R.Z.); cinzia.sada@unipd.it (C.S.) \\ 3 Institute of Applied Physics, University of Muenster, 48159 Münster, Germany \\ 4 Institute of Applied Sciences and Intelligent Systems, CNR, 80078 Pozzuoli Napoli, Italy \\ 5 Physics Faculty, Taras Shevchenko National University of Kyiv, 01601 Kyiv, Ukraine; \\ VReshetnyak@univ.kiev.ua \\ * Correspondence: f.simoni@univpm.it
}

Citation: Ciciulla, F.; Zaltron, A.; Zamboni, R.; Sada, C.; Simoni, F.; Reshetnyak, V.Y.; Lucchetti, L. Optofluidic Platform Based on Liquid Crystals in X-Cut Lithium Niobate: Thresholdless All-Optical Response. Crystals 2021, 11, 908. https:// doi.org/10.3390/cryst11080908

Academic Editors: Reshef Tenne, Zoran Radić, Anna Moliterni, Robert F. Klie, Rocco Caliandro and Dritan Siliqi

Received: 6 July 2021

Accepted: 29 July 2021

Published: 2 August 2021

Publisher's Note: MDPI stays neutral with regard to jurisdictional claims in published maps and institutional affiliations.

Copyright: (c) 2021 by the authors. Licensee MDPI, Basel, Switzerland. This article is an open access article distributed under the terms and conditions of the Creative Commons Attribution (CC BY) license (https:/ / creativecommons.org/licenses/by/ $4.0 /)$.

\begin{abstract}
In this study, we present a new configuration of the recently reported optofluidic platform exploiting liquid crystals reorientation in lithium niobate channels. In order to avoid the threshold behaviour observed in the optical control of the device, we propose microchannels realized in a x-cut crystal closed by a z-cut crystal on the top. In this way, the light-induced photovoltaic field is not uniform inside the liquid crystal layer and therefore the conditions for a thresholdless reorientation are realized. We performed simulations of the photovoltaic effect based on the well assessed model for Lithium Niobate, showing that not uniform orientation and value of the field should be expected inside the microchannel. In agreement with the re-orientational properties of nematic liquid crystals, experimental data confirm the expected thresholdless behaviour. The observed liquid crystal response exhibits two different regimes and the response time shows an unusual dependence on light intensity, both features indicating the presence of additional photo-induced fields appearing above a light intensity of $10^{7} \mathrm{~W} / \mathrm{m}^{2}$.
\end{abstract}

Keywords: lithium niobate; liquid crystals; threshold-less response; photovoltaic; optofluidics

\section{Introduction}

Lithium niobate $\left(\mathrm{LiNbO}_{3}\right)$ is among one of the most used crystalline materials in photonic applications [1-3] and has been recently proposed as an optically active substrate for the realization of microfluidic devices [4,5], opening its use in the field of optofluidics. Additionally, liquid crystals (LC) are well-known to be very sensitive to external stimuli, such as optical and electric fields, making them attractive for photonic applications beside their large use for different types of displays [6]. Recently, the possibility of combining these two materials for advanced all-optical devices was demonstrated [7-9]. In these investigations, a key role is played by the bulk photovoltaic effect induced by light in $\mathrm{LiNbO}_{3}$ crystals $[10,11]$ and successfully used to reorient the molecular LC director in properly designed nematic liquid crystal cells [7-9,12]. The bulk photovoltaic effect consists in the appearance of a photo-induced current generated in $\mathrm{LiNbO}_{3}$ upon light illumination. The subsequent charge redistribution that takes place inside the crystal gives rise to an electric field, the photovoltaic field [11], that has been exploited to realize photorefractive optics in $\mathrm{LiNbO}_{3}[13,14]$. This effect is strongly enhanced by doping $\mathrm{LiNbO}_{3}$ with iron $[10,15]$, which introduces electron donor $\left(\mathrm{Fe}^{2+}\right.$ ions) and acceptor $\left(\mathrm{Fe}^{3+}\right.$ ions) centers and makes it possible to reach a photovoltaic field up to $10^{7} \mathrm{~V} / \mathrm{m}$.

The combination of $\mathrm{LiNbO}_{3}$ and $\mathrm{LC}$ has also been investigated in microfluidic configuration [16], and all optical control of the LC orientation in microfluidic channels engraved 
in iron-doped $\mathrm{LiNbO}_{3}$ crystals has been demonstrated. In the configuration of Ref. [16], the microchannels were obtained in two z-cut crystals and the light-induced electric field was orthogonal to the crystal surfaces. Since the initial LC orientation was along the microfluidic channel direction, the director reorientation showed a threshold for the field in a way similar to the conventional Freedericks transition occurring in planar aligned nematic LC.

A typical configuration in LC technology is the hybrid aligned nematic cell (HAN) realized with planar alignment on one boundary and homeotropic alignment on the other, showing no re-orientational threshold both to electric and optical fields [17-19]. This behavior is due to the initial director alignment that is found neither orthogonal nor parallel to the electric field along most of the sample thickness even at low field values. In case of planar alignment of LC, a similar configuration can in principle be realized if the applied electric field has a hybrid configuration, i.e., it is not uniformly aligned along the sample thickness. Such a geometry can be realized if the field is orthogonal to the boundary on one surface and parallel to the boundary on the other.

In order to realize a similar configuration, we built a microfluidic chip based on two $\mathrm{LiNbO}_{3}$ crystals with different crystallographic orientations: a x-cut crystal used as the chip platform where we dug the microchannel and a z-cut crystal used as a cover closing the fluidic channel on the upper side. A single z-cut crystal would give rise to a light-induced electric field having components perpendicular and parallel to the crystal surface, the first component being higher than the second. Such a configuration has been recently proved to affect the LC orientation giving rise to topological defects able to generate optical vortex [20]. The use of an additional $x$-cut crystal as a host for the fluidic channel strengthens the inplane component of the photovoltaic field, since it gives rise to a field parallel to the surface. Specifically, light illumination of the x-cut crystal produces a charge accumulation on both the lateral walls and the bottom of the fluidic channel, thus originating fields mainly oriented in plane. Since the alignment of the LC inside the microchannel is approximately planar with the director oriented along the channel length, with these two $\mathrm{LiNbO}_{3}$ crystals one can realize the conditions for a hybrid-oriented photo-induced field. Results show that a threshold-less LC reorientation is indeed obtained, a behavior interesting for optofluidic applications, where the development of active or passive optical devices based on a proper material is currently the subject of wide investigation. The observed threshold-less LC actuation combined with the recently observed threshold LC response [16] constitute a bundle of strategies in the frame of the possible generation of all optical devices based on $\mathrm{LiNbO}_{3}$ optofluidic platforms.

The device is realized by engraving the microfluidic channel in a x-cut $\mathrm{LiNbO}_{3}: \mathrm{Fe}$ crystal and using a flat $\mathrm{LiNbO}_{3}: \mathrm{Fe}$ z cut crystal as the top boundary closing the channel on one side. In this paper we give a detailed study of the proposed configuration, demonstrating the expected threshold-less behavior of the opto-optical response and reporting a response time about one order of magnitude shorter than in the previously investigated configuration.

In Section 2 we provide the description of the experimental details; simulations of the expected profile of the fields generated by light irradiation in the lithium niobate optofluidic platform are reported in Section 3; Section 4 is devoted to presentation and discussion of the experimental results followed by concluding remarks.

\section{Experimental Procedure}

The $\mathrm{LiNbO}_{3}: \mathrm{Fe}$ crystals to be used as substrates of the liquid crystals microfluidic chip have been obtained from a boule grown by the Czochralski technique having congruent composition with a dopant concentration $\mathrm{CFe}_{\mathrm{Fe}}=18.8 \times 10^{18} \mathrm{at} / \mathrm{cm}^{3}(0.1 \% \mathrm{~mol})$. The boule was poled in air above the Curie temperature at $1200^{\circ} \mathrm{C}$, in order to get a single domain structure that was checked by X-ray Diffraction. Then, it was oriented along the three crystallographic axes of the material and cut in order to get samples with the main faces perpendicular (z-cut crystal) or parallel (x-cut crystal) to the c-axis of lithium niobate. Both crystals were polished by means of a Logitech PM5 lapping machine in order to get good 
optical quality of the main surfaces. Finally, the as-grown x-cut crystal that will act as the microfluidic platform was characterized by means of optical absorption measurements [21] to calculate its reduction degree $\mathrm{R}=\mathrm{Fe}^{2+} / \mathrm{Fe}^{3+}$, which is equal to $\mathrm{R}_{\mathrm{x}}=0.010 \pm 0.003$, whereas the $\mathrm{z}$ cut crystal underwent a reduction thermal treatment at $500{ }^{\circ} \mathrm{C}$ in a gas mixture of $\operatorname{Ar}(98 \%)+\mathrm{H}_{2}(2 \%)$, leading a to a final reduction degree of $\mathrm{R}_{\mathrm{z}}=0.008 \pm 0.002$.

An open channel with rectangular cross section $(200 \mu \mathrm{m}$ [width] $\times 100 \mu \mathrm{m}$ [height] $)$ has been realized on the surface of the $x$-cut crystal using a precision polymeric saw coated with diamond particles for the mechanical micromachining, which allows obtaining a roughness of the lateral surfaces of the microchannel of few $\mathrm{nm}$ [22]. In this way, the channel length is parallel to the optical axis (c-axis) of the $\mathrm{x}$-cut substrate, whereas the lateral walls and the bottom are perpendicular to its $y$-axis and to its $x$-axis, respectively. The choice of a x-cut crystal as a platform for the optofluidic circuit allows to minimize the vertical component of the light-induced electric field in this substrate, indeed the components of the photovoltaic matrix along the x-axis vanish, independently on the light polarization direction [2]. In this way, on the bottom of the microchannel a charge accumulation can be observed only at the border of the beam light. Worthy of note, the use of a light beam large enough to illuminate the lateral walls would give rise to charge accumulation also in these regions.

The microfluidic chip is finally realized by closing the open side of the channel by the flat z-cut crystal and filling it with the nematic liquid crystal E7 (Merck) using a special syringe connector. A sketch of the microfluidic chip is shown in Figure 1a, together with a polarizing optical microscope image of the filled microchannel (1b).

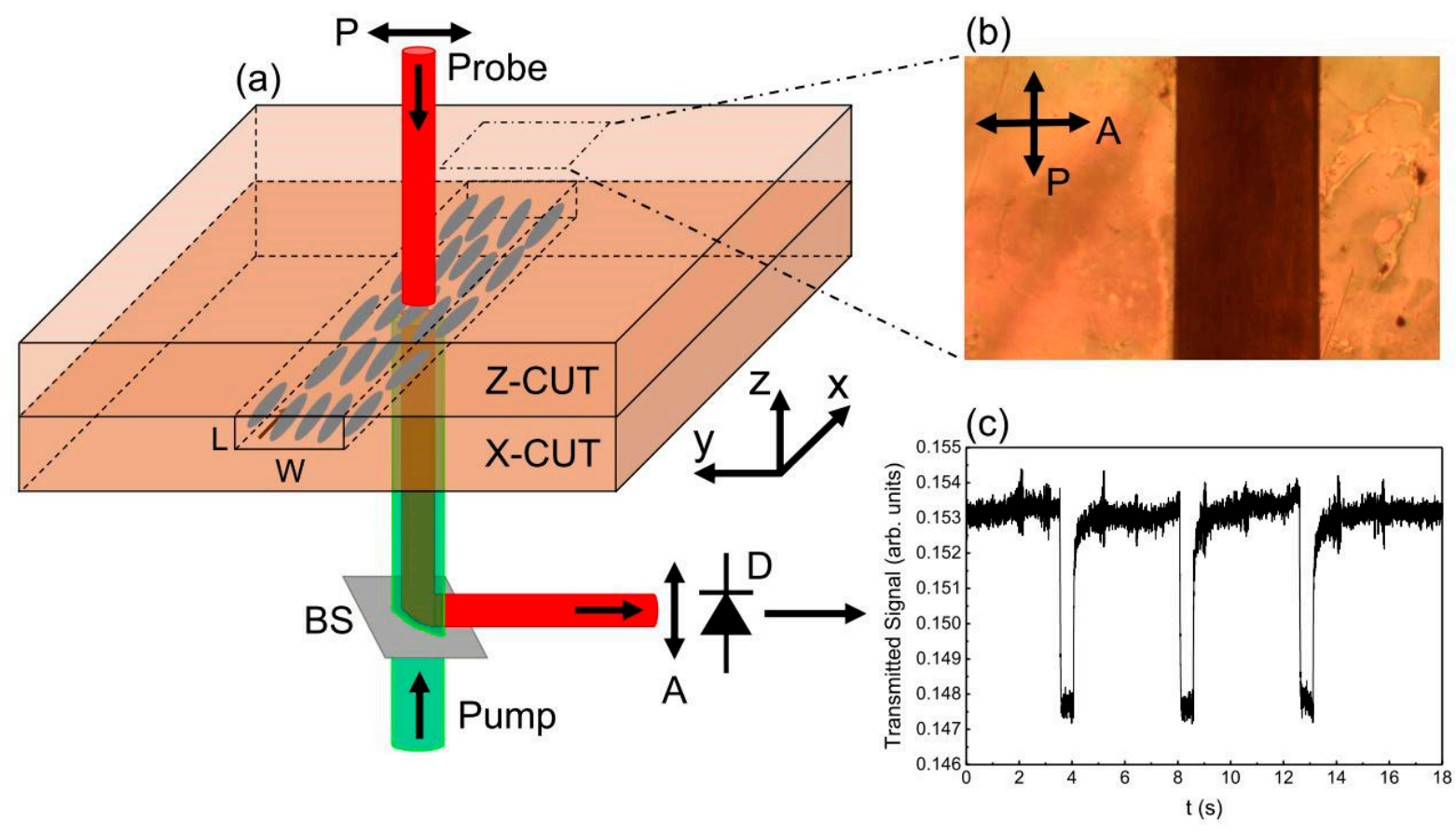

Figure 1. Experimental details. (a) Sketch of the microfluidic chip with the pump and probe beams counterpropagating in the LC-filled microchannel along z; (b) polarizing optical microscope image of the microchannel filled with E7. The axis of the microscope polarizer is parallel to the channel length; (c) example of the raw data observed upon pump irradiation with $500 \mathrm{~ms}$ cycles separated by $2 \mathrm{~s}$ of dark. The graph corresponds to a pump intensity $\mathrm{I}=7 \times 106 \mathrm{~W} / \mathrm{m}^{2}$.

The image in Figure 1b is taken with the axis of the polarizer parallel to the channel length, while keeping the analyzer crossed. The LC alignment is planar and uniform except for regions close to the walls, where defects (not shown) are induced by the channel geometry. The LC is at rest in all the experiments reported here. 
The optical response of the optofluidic chip has been investigated by using a conventional pump-probe technique: the pump beam is provided by the green line of a cw $\mathrm{Ar}$ ion laser $(\lambda=514 \mathrm{~nm})$ focused to a waist of $30 \mu \mathrm{m}$ in correspondence to the microchannel central region, where it impinges at normal incidence. The pump light is linearly polarized parallel to the microchannel axis and its power varies in the range $0-50 \mathrm{~mW}$, which leads to a pump intensity $I$ in the range $0-1.8 \times 10^{7} \mathrm{~W} / \mathrm{m}^{2}$. A mechanical shutter enabled performing irradiation cycles, which in the experiments reported here had $500 \mathrm{~ms}$ duration and $2 \mathrm{~s}$ dark time separation. The probe beam originates from a low-power He-Ne laser $(\lambda=633 \mathrm{~nm})$ focused to a waist of $20 \mu \mathrm{m}$ and counter propagating with respect to the green pump beam. Probe polarization is linear and forms an angle of $45^{\circ}$ with that of the pump. The probe light transmitted by the sample, orthogonally polarized with respect to the incident one, was detected by a photodiode connected to a computer. Pump irradiation was performed on the $\mathrm{x}$-cut side of the optofluidic chip. Since the pump beam waist is significantly smaller than the width of the fluidic channel, the charge accumulation at the lateral walls can be neglected. A scheme of the pump and probe beams impinging on the LC-filled microchannel is reported in Figure 1a.

In the configuration described above, light propagates orthogonal to the $\mathrm{c}$ axis of the $\mathrm{x}$-cut chip platform and along the $\mathrm{c}$ axis of the z-cut cup. Being linearly polarized in the cell plane, light does not undergo any phase shift due to the z-cut crystal birefringence, while it can be affected by propagation along the x-cut crystal. In order to take into account this latter possible contribution and separate it from the signal due to LC reorientation, pump-probe measurements were also performed before filling the microchannel with the LC.

As previously described $[7,16]$, due to the dependence of the detected signal on the phase shift seen by the probe light travelling through the LC-filled region, the experimental data can easily be related to the birefringence $\Delta \mathrm{n}$ induced in the LC which, on its turn, is a signature of molecular reorientation [23].

\section{Calculation of the Light-Induced Electric field}

In order to calculate the light-induced electric field profile inside the LC layer bounded by two different lithium niobate substrates, we consider irradiation by light having a Gaussian profile of the intensity. The light illumination creates surface charges located at the top and bottom surfaces of the z-cut substrate, whereas the $x$-cut substrate contributes with the presence of in-plane light-induced electric fields localized on the bottom of the fluidic channel. To find the electric field spatial profile one needs to solve the Poisson equation:

$$
\nabla \cdot\left(\varepsilon_{0} \hat{\varepsilon} \mathbf{E}\right)=\rho(\mathbf{r}),
$$

in x-cut and z-cut in the $\mathrm{LiNbO}_{3}$ substrates and in the LC layer. In Equation (1), $\hat{\varepsilon}$ is the dielectric tensor and $\rho(\mathbf{r}, \mathbf{z})$ is the charge density in the corresponding layers.

The surface charge density in the z-cut crystal is given by

$$
\rho_{\text {surf }}=\rho_{0, \text { surf }} \exp \left(-\frac{1}{2}\left[\frac{r}{\omega}\right]^{2}\right)
$$

where $\omega$ is the light beam waist and the magnitude of the surface charge density at saturation is $\rho_{0, \text { surf }}=10^{-2} \mathrm{C} / \mathrm{m}^{2}$

We further assume that the volume free charges are only present in $\mathrm{x}$-cut substrate and to estimate them we proceed as follows. Under light illumination the free charges (electrons) are generated from the donors in $\mathrm{LiNbO}_{3}$. The rate equation for excitation of electron donors, $N_{D}^{*}$, takes the form [11,24-27]:

$$
\frac{\partial N_{D}^{*}}{\partial t}=\left[\frac{\sigma}{h v} I_{0} \exp \left(-r^{2} / 2 \omega^{2}\right)+\beta\right]\left(N_{D}-N_{D}^{*}\right)-\gamma n N_{D}^{*},
$$


where $N_{D}$ is the concentration of electron donors, $\sigma$ is donors photoionization cross section, $\beta$ is the thermal excitation rate, $I_{0}$ is the light intensity, $\gamma$ is the recombination rate, and $n(\mathbf{r})$ is the electrons density.

The stationary solution to this equation is:

$$
N_{D}^{*}(\mathbf{r})=\frac{N_{D}\left[\frac{\sigma}{h v} I_{0} \exp \left(-\frac{r^{2}}{2 \omega^{2}}\right)+\beta\right]}{\beta+\gamma n(\mathbf{r})+\frac{\sigma}{h v} I_{0} \exp \left(-\frac{r^{2}}{2 \omega^{2}}\right)},
$$

The volume concentration of charges is given by $\rho(\mathbf{r})=e\left(N_{D}^{*}(\mathbf{r})-n(\mathbf{r})-N_{A}\right) . N_{A}$ is the concentration of charges that compensate for the charge of $N_{D}^{*}$ under dark conditions ( $N_{A}$ is the concentration of acceptor centers). Therefore, the charges conservation equation (continuity equation) reads:

$$
e \frac{\partial}{\partial t}\left(N_{D}^{*}(\mathbf{r})-n(\mathbf{r})-N_{A}\right)+\nabla \cdot \mathbf{j}(\mathbf{r})=0,
$$

here, $\mathbf{j}(\mathbf{r})$ is the current density. Assuming $N_{A}=$ const, the continuity equation simplifies to:

$$
e \frac{\partial}{\partial t}\left(N_{D}^{*}(\mathbf{r})-n(\mathbf{r})\right)+\nabla \cdot \mathbf{j}(\mathbf{r})=0
$$

The electric current has three contributions, the first one originates from the electrons motion in the electric field (electrons drift), the second one comes from the diffusion due to the gradient of the electrons concentration and the third one is the photovoltaic current.

Then the total current density is given by:

$$
\mathbf{j}=e \mu n \mathbf{E}+e D_{e} \nabla n+\alpha k_{G} I_{0} \exp \left(-\frac{r^{2}}{2 \omega^{2}}\right) \mathbf{e}_{c}
$$

where $\mathbf{e}_{c}$ is the unit vector of c-axis.

Since $\mathbf{D}=\varepsilon_{0} \hat{\varepsilon} \mathbf{E}$ the equation for the displacement reads:

$$
\nabla \times \mathbf{D}=e\left(N_{D}^{*}(\mathbf{r})-n(\mathbf{r})-N_{A}\right) .
$$

In the open-circuit case $\mathbf{j}=0$, then according to Equation (7) we have:

$$
\mathbf{E}=-\frac{D_{e}}{\mu n} \nabla n-\frac{1}{e \mu n} \alpha k_{G} I_{0} \exp \left(-\frac{r^{2}}{2 \omega^{2}}\right) \mathbf{e}_{c} m
$$

where $D_{e}$ is the electron diffusion coefficient in $\mathrm{LiNbO}_{3}$ and $\mu$ is the electron mobility.

We neglect the contribution of field originated in the z-cut substrate onto the electric field (and charges profile) in the $x$-cut substrate. In this way, multiplying both sides of the Equation (9) by $\varepsilon_{0} \hat{\varepsilon}_{L N}$ and using Equation (8) we obtain the equation for the charges concentration $n(\mathbf{r})$ in $\mathbf{x}$-cut substrate:

$$
\nabla \cdot\left[\frac{\varepsilon_{0} \hat{\varepsilon}_{L N} D_{e}}{\mu n(\mathbf{r})} \nabla n(\mathbf{r})+\frac{\varepsilon_{0} \hat{\varepsilon}_{L N}}{e \mu n(\mathbf{r})} \alpha k_{G} I_{0} \exp \left(-\frac{r^{2}}{2 \omega^{2}}\right) \mathbf{e}_{c}\right]=-e\left(N_{D}^{*}(\mathbf{r})-n(\mathbf{r})-N_{A}\right),
$$

where the concentration of the excited donors $N_{D}^{*}(\mathbf{r})$ is given by Equation (4).

After solving Equation (10) numerically and finding the electron concentration spatial profile $n(\mathbf{r})$, Equation (8) can be used for calculating the electric field in the LC slab due to the $x$-cut substrate. On the other hand, the contribution of the z-cut substrate to the field in the LC layer is given by using the surface charge distribution given by Equation (2). The total field is then given by the sum of the light-induced fields in the $\mathrm{x}$-cut and in the $\mathrm{z}$-cut substrates.

In Figure 2 the components $x$ and $z$ of the electric field are reported as a function of the distance from the beam axis located at $x=0$, as calculated in the middle of the LC 
layer ( $\mathrm{z}=L / 2, L$ being the height of the microchannel, i.e., the thickness of the $L C$ layer). In Figure 3 the same components are plotted as a function of $z$, i.e., through the LC layer, both at the center of the beam ( $a$ and $b$ ) and at a distance $x=100 \mu \mathrm{m}$ from the beam axis (c and d).

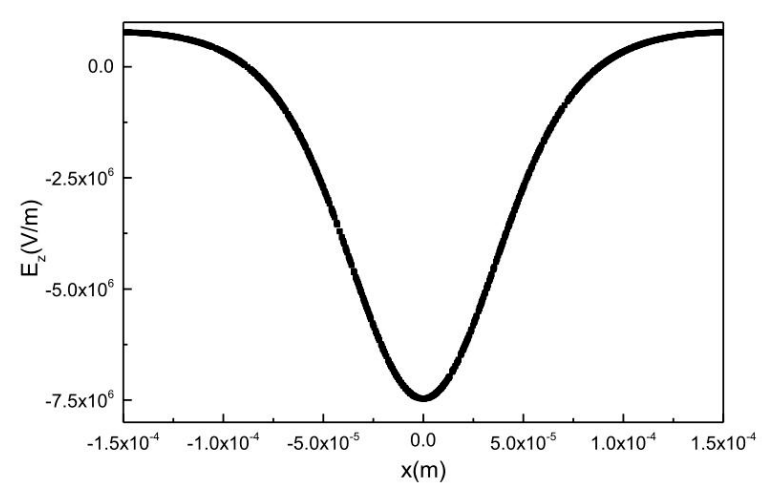

(a)

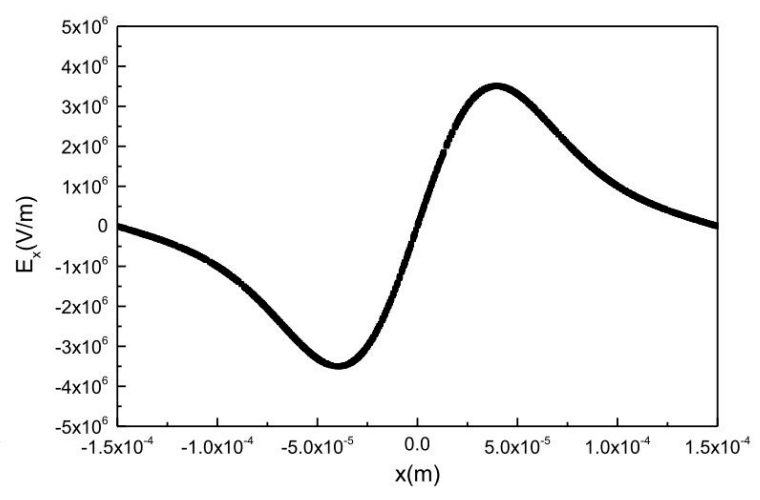

(b)

Figure 2. Light-induced electric field components in correspondence of the middle of the LC layer $(\mathrm{z}=\mathrm{L} / 2)$ as a function of the distance $x$ from the peak of the Gaussian beam waist. The used data are: LC: thickness $50 \mu \mathrm{m}, \varepsilon_{\mathrm{e}}=14.5, \varepsilon_{\mathrm{o}}=5.1$; $\mathrm{LiNbO}_{3}$ : thickness $900 \mu \mathrm{m}, \varepsilon_{\mathrm{e}}=29, \varepsilon_{\mathrm{O}}=85$. (a) Field component perpendicular to the substrate; (b) field component parallel to the substrate.

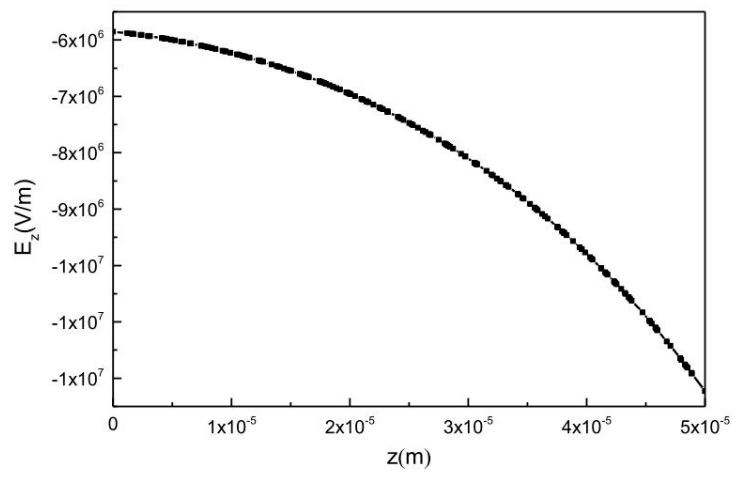

(a)

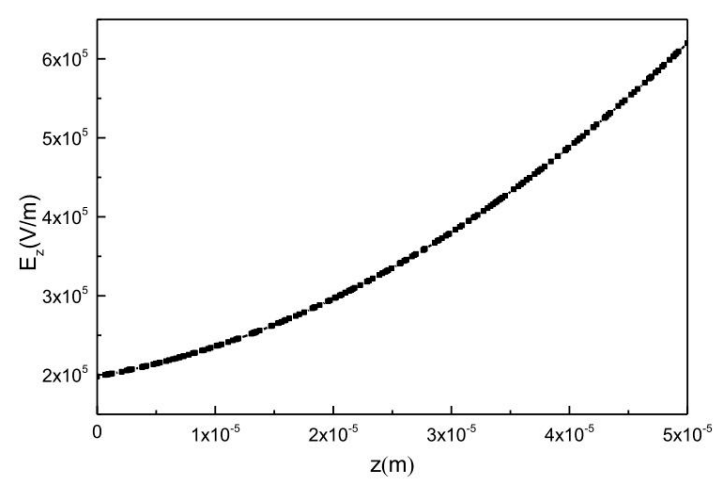

(c)

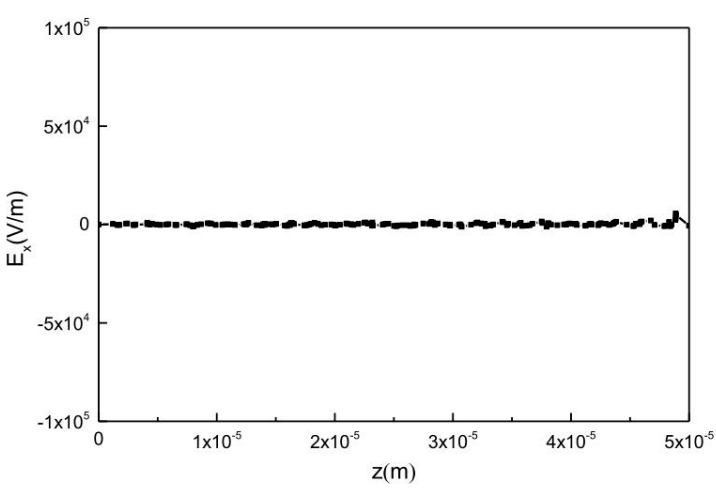

(b)

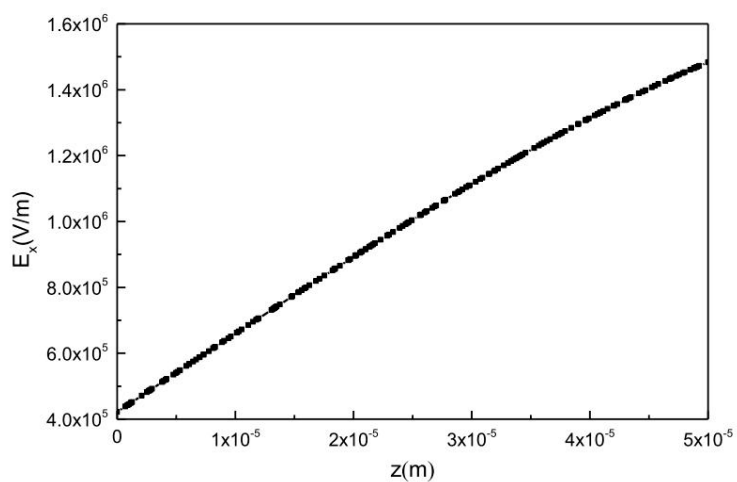

(d)

Figure 3. Light-induced electric field components vs z, showing the profile of the electric field through the LC thickness. (a) $\mathrm{z}$ component perpendicular to the substrates and $(\mathbf{b}) \mathrm{x}$ component parallel to the substrates at the center of the beam $\mathrm{x}=0$; (c) and (d) $z$ component and $x$ component at a distance $100 \mu \mathrm{m}$ from the beam center. Used data as in Figure $2 . \mathrm{z}=0$ is located on the $\mathrm{x}$-cut crystal surface in contact with the LC layer, $\mathrm{z}=\mathrm{L}$ is located on the $\mathrm{z}$-cut crystal surface in contact with the LC layer. 
In Figure 2 we observe that, at $z=L / 2$, the radial and the transversal components $E_{z}$ of the electric field have a strong variation in the laser waist area and are of the same order of magnitude in many points, therefore they originate an oblique total electric field. Note that the behavior of the other in-plane component $E_{y}$ is similar to the one calculated for $E_{x}$. Figure 3 shows that the in-plane component of the photovoltaic field is zero along the LC layer only right on the beam axis, while it is comparable to the $\mathrm{z}$-component elsewhere and thus it affects the direction of the total electric field in the LC bulk.

The expected "hybrid" orientation of the light-induced electric field is reported in Figure 4 where the field profile is represented by arrows. Arrows lengths are proportional to the field strength in Figure $4 \mathrm{a}$ and are represented in logarithmic scale in Figure $4 \mathrm{~b}$. The center of the slab corresponds to the peak of the light beam intensity.

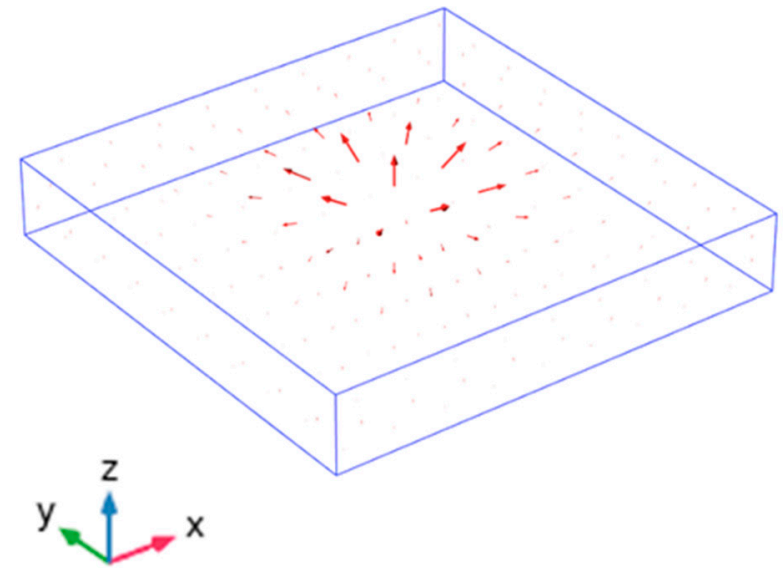

(a)

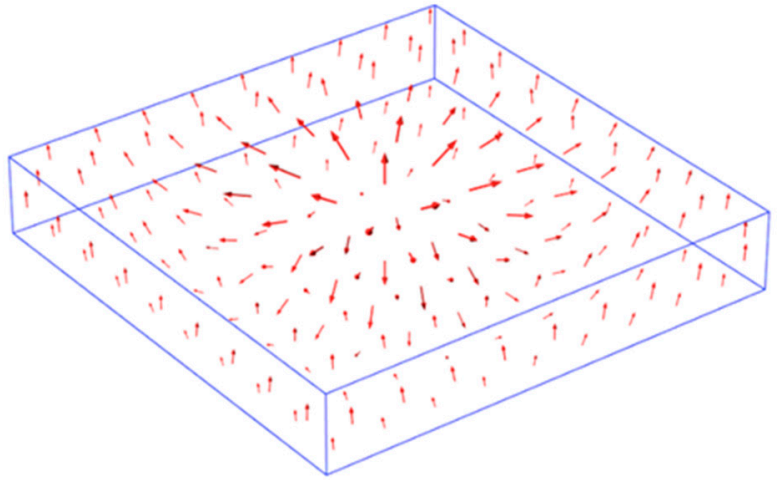

(b)

Figure 4. Overview of the profile of the light-induced electric field distribution inside the liquid crystal layer in the optofluidic channel. (a) Length of the arrows proportional to the field strength; (b) same as (a) in logarithmic scale, made in order to make the spatial evolution of the electric field direction inside the liquid crystal layer more visible.

\section{Results and Discussion}

Pump irradiation of the LC filled microchannel leads to a decrease of the probe transmission for each value of the used power, as shown in the inset of Figure 1c where a typical example of the signal detected is reported for a single value of the pump intensity $I$ $\left(I=7 \times 10^{6} \mathrm{~W} / \mathrm{m}^{2}\right)$.

From these and similar data we determined both the amplitude of the transmission variation $\Delta \mathrm{T}$ and the response times (on and off) as a function of the impinging pump intensity $I . \Delta \mathrm{T}$ vs. $I$ is shown in Figure 5, where the threshold-less character of the LC response is evident. This behavior is in agreement with the profile of the photoinduced space charge field reported in Figure 4, which suggests the formation of a hybrid-oriented photo-induced field, which is indeed able to affect the LC molecular orientation without the need for any threshold value to be overcome, as expected. This behavior is due to the mutual direction of the light-induced electric field and the initial director configuration, that is neither 0 nor $\pi / 2$ along most of the sample thickness and mimics the configuration of a hybrid LC cell, that is characterized by threshold-less molecular reorientation. The amount of probe transmitted light between crossed polarizers is related to the LC birefringence $\Delta \mathrm{n}$ through the induced phase shift $\delta[24]: \mathrm{I}_{\mathrm{T}}=\mathrm{I}_{0} \sin ^{2}(\delta / 2)$, where $\delta=2 \pi \Delta \mathrm{n} / \lambda$. Therefore, $\mathrm{a}$ measurement of $\Delta \mathrm{T}$ also gives a way to evaluate the LC distortion induced by the action of the optically-generated electric field. 


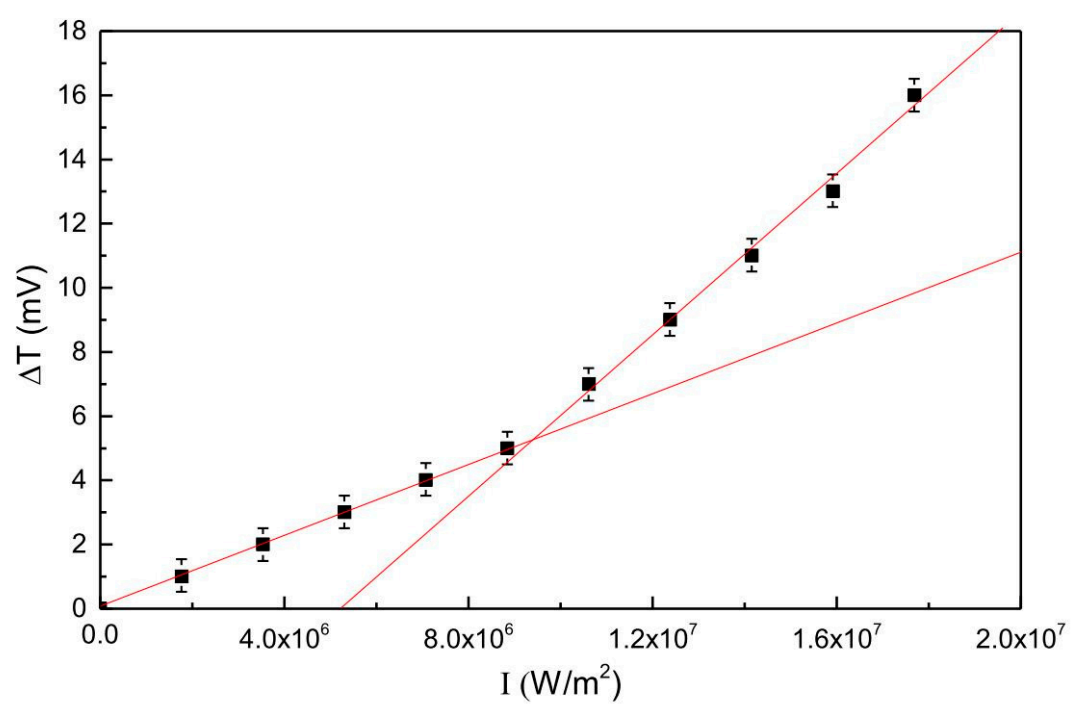

Figure 5. Transmitted probe signal vs. pump light intensity. A threshold-less response of the LC molecular director is clearly observed. The curve slope changes in correspondence of a light intensity $I=1.1 \times 10^{7} \mathrm{~W} / \mathrm{m}^{2}$.

Interestingly, $\Delta \mathrm{T}$ exhibits a linear dependence on $I$ for values lower than $I=1.1 \times$ $10^{7} \mathrm{~W} / \mathrm{m}^{2}$, then, the slope of the curve changes and becomes steeper, indicating that a higher field is responsible for the LC reorientation in this range of intensity. The observed linear dependence of the signal on the pump intensity for $I<1.1 \times 10^{7} \mathrm{~W} / \mathrm{m}^{2}$ deserves some comment. According to the one center model [19], valid up to about $10^{7} \mathrm{~W} / \mathrm{m}^{2}$, the saturation value of the optically induced photovoltaic field in iron doped $\mathrm{LiNbO}_{3}$ crystals is independent on light intensity, thus an intensity independent response would be expected for intensity values lower than the one mentioned above. However, the time $\tau$ the photovoltaic field needs to reach the saturation value, does depend on $I$ and it decreases with increasing intensity. Specifically, the relation between $\tau$ and $I$ has the form $[11,24]$ :

$$
\tau=\frac{\varepsilon \varepsilon_{0}}{\sigma_{p h}+\sigma_{d}}=\frac{\varepsilon \varepsilon_{0}}{e \mu s q} \frac{\gamma h v}{I R} \cong \frac{510^{4}}{I R}
$$

where $\sigma_{p h}$ and $\sigma_{d}$ are the photo and dark conductivities, $\varepsilon$ is the proper dielectric constant of $\mathrm{LiNbO}_{3}, \gamma$ is the recombination coefficient, $q$ is the quantum efficiency of excitation of an electron upon absorption of a photon, $\mu$ is the carrier mobility, $s$ is the photon absorption cross section, and $R$ the reduction degree [24]. In Equation (11) the constant value $5 \times 10^{4} \mathrm{~J} / \mathrm{m}^{2}$ takes into account all the indicated parameters. In this way, knowing the reduction factor of the crystal it is possible to derive the value of $\tau$ for each value of the intensity $I$. This is shown in Figure 6 for $I$ in the range $\left(0 \div 9 \times 10^{6}\right) \mathrm{W} / \mathrm{m}^{2}$ and for $R=0.01$, the reduction degree of the crystals used to build the substrate of the optofluidic chip. With this value of $R, \tau$ is longer than the irradiation time for all the values of $I$, thus the field does not reach saturation during light irradiation for any of the used values of the pump intensity. This means that each value of I corresponds to a different value of the photovoltaic field, all the values being lower than the saturation, which explains the observed intensity dependent response. 


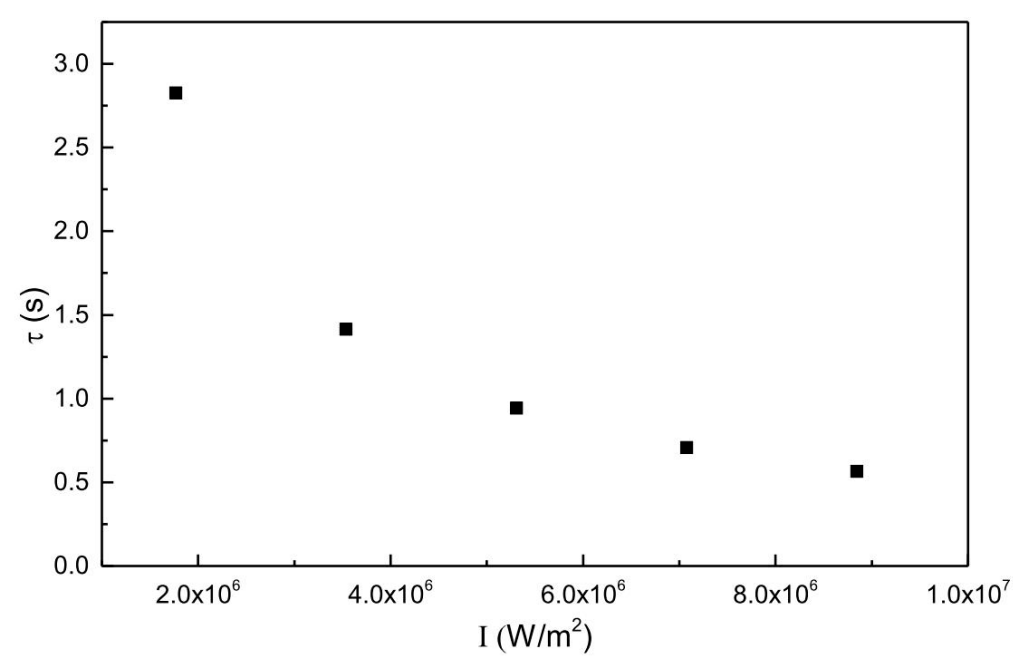

Figure 6. Photovoltaic time constants as function of the pump light intensity in the range $\left(0-9 \times 10^{6}\right) \mathrm{W} / \mathrm{m}^{2}$, i.e., in the range of intensities before the change of slope of the curve $\Delta \mathrm{T}$ vs. I (see Figure 5).

As already mentioned, the one center model is valid up to light intensity on the order of $\mathrm{I} \sim 1 \times 10^{7} \mathrm{~W} / \mathrm{m}^{2}$ and the photovoltaic field is expected to increase and to become intensity dependent for higher values of $I[11,24]$. The dependence on $I$ and the slope variation observed in the second part of the curve in Figure 5 are thus easily explained; an additional contribution can also come from the pyroelectric field, which cannot be excluded at these intensity values [16].

Measurements performed before filling the microchannel with E7 gave a transmission variation lower by at least one order of magnitude with respect to the one observed when the LC is present, and independent of the pump light intensity. This indicates that the transmission variation $\Delta \mathrm{T}$ reported in Figure 5 is only due to the action of the electric field generated by light in lithium niobate on the LC average molecular orientation.

The response times have been evaluated by exponential best fits of the rise and decay portions of the detected signal. The decay time $\tau_{\text {off }}$ is of the order of $100 \mathrm{~ms}$, similar to what is observed in [16], while the rise time $\tau_{o n}$ appears shorter. It is reported in Figure 7 as a function of the pump intensity $I$. Two comments have to be made about $\tau_{o n}$ : (i) it decreases linearly with increasing $I$ up to $I=1.1 \times 10^{7} \mathrm{~W} / \mathrm{m}^{2}$, a behavior in agreement with the relation [28] $\tau_{o n} \approx \frac{\gamma}{\varepsilon_{0}|\Delta \varepsilon| E^{2}}$ describing the rise time for molecular reorientation of a nematic LC with viscosity $\gamma$ and dielectric anisotropy $\Delta \varepsilon$. On the other hand, the actual values of $\tau_{o n}$ are orders of magnitude lower than those expected; (ii) as observed for $\Delta \mathrm{T}$, the curve changes in correspondence of $I=1.1 \times 10^{7} \mathrm{~W} / \mathrm{m}^{2}$; in particular $\tau_{o n}$ starts increasing with increasing $I$. These two peculiar features are not easily accounted for. The fast dynamics could indicate that the LC reorientation involves only a thin layer of the whole sample, as already observed in [16], however the reasons for this cannot be directly related to any particular characteristic of the analyzed microfluidic system. The behavior observed above $1.1 \times 10^{7} \mathrm{~W} / \mathrm{m}^{2}$ is even more puzzling and may be a further indication of the appearance of additional contributions to the photo-induced fields, namely the pyroelectric field or even the field generated by the channel walls that may come into play at high intensity. This, due to a convolution of the different stimuli, may give rise to a different dependence of the response time on $I$. Further investigations are in progress for clarifying these experimental observations. 


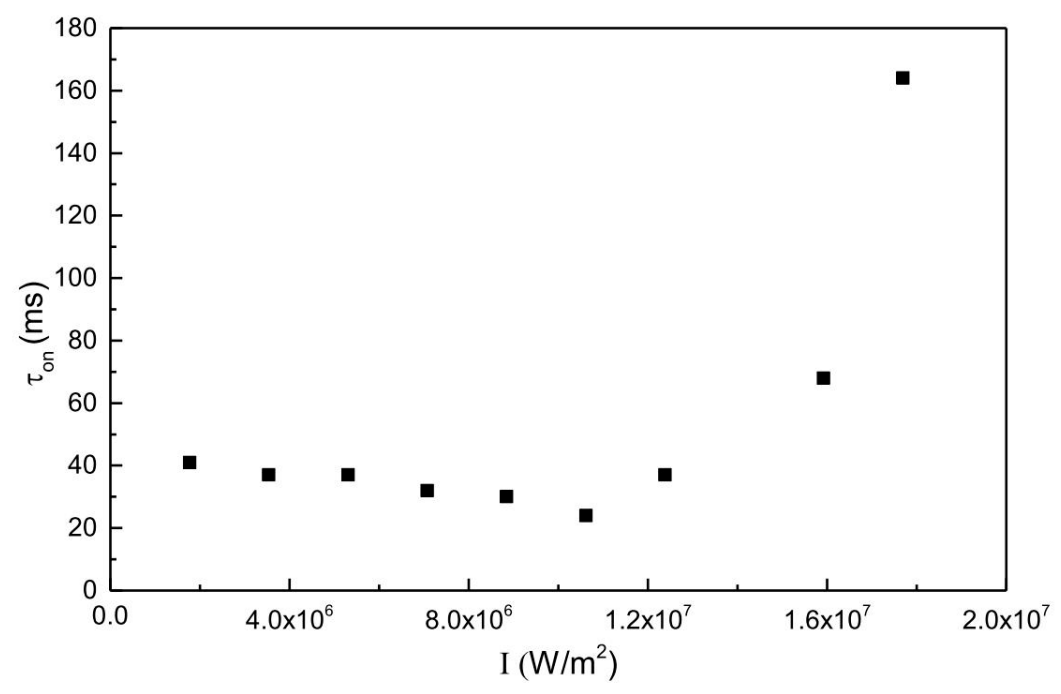

Figure 7. Rise time $\tau_{\text {on }}$ as evaluated from exponential fits of the raw data as a function of the pump light intensity. A change of the dependence on I is observed for values of light intensity higher than $1.1 \times 10^{7} \mathrm{~W} / \mathrm{m}^{2}$.

\section{Conclusions}

Combining two lithium niobate crystals with different crystallographic orientation, we demonstrated that a threshold-less all optical control of an optofluidic LC chip can be realized. Both numerical simulations and experimental data confirm that the observed behavior is due to the not uniform distribution of the field in the LC layer.

Besides being thresholdless, the LC response exhibits two different regimes, with a change of slope above a specific intensity; moreover, the LC response time is faster than expected and shows an unusual dependence on light intensity. These features, although not yet completely understood, seem to indicate the presence of additional photo-induced contributions that appear for values of I above $10^{7} \mathrm{~W} / \mathrm{m}^{2}$.

The reported threshold-less LC actuation together with the recently observed threshold LC response [16] constitute a body of strategies in the frame of the possible development of all optical devices based on lithium niobate optofluidic platforms.

Author Contributions: Conceptualization, L.L., F.S., A.Z.; preparation of $\mathrm{LiNbO}_{3}$ crystals, A.Z., R.Z., C.S.; theory and simulation V.Y.R.; performing optical measurements F.C., L.L.; analyzing data L.L., F.S.; writing and editing L.L., F.S., V.Y.R., A.Z. All authors have read and agreed to the published version of the manuscript.

Funding: This work has been partially funded by the project BIRD165523/16 "Biosensing Lightdriven Tools in Advanced opto-microfluidic Lithium niobate Platform" and by the Excellence Project "Integrated Opto-Microfluidic Prototype on Lithium Niobate Crystals for Sensing Applications" (call 2011-2012) founded by Ca.Ri.Pa.Ro foundation.

Acknowledgments: C.S., A.Z. and R.Z. kindly acknowledge M. Chauvet and the FEMTO-ST Institute (Besancon, France) for their help in realizing the microfluidic channels in the lithium niobate substrate.

Conflicts of Interest: The authors declare no conflict of interest.

\section{References}

1. Arizmendi, L. Photonic applications of lithium niobate crystals. Phys. Status Solidi 2004, 201, 253-283. [CrossRef]

2. Weis, R.S.; Gaylord, T.K. Lithium niobate: Summary of physical properties and crystal structure. Appl. Phys. A Mater. Sci. Process. 1985, 37, 191-203. [CrossRef]

3. Wong, K.K. Properties of Lithium Niobate; INSPEC: London, UK, 2002.

4. Zaltron, A.; Bettella, G.; Pozza, G.; Zamboni, R.; Ciampolillo, M.V.; Argiolas, N.; Sada, C.; Kroesen, S.; Esseling, M.; Denz, C. Integrated optics in lithium niobate crystals for sensing applications. Proc. SPIE 2015, 9506, 950608. 
5. Gazzetto, M.; Nava, G.; Zaltron, A.; Cristiani, I.; Sada, C.; Minzioni, P. Numerical and experimental study of optoelectronic trapping on iron-doped lithium niobate substrate. Crystals 2016, 6, 123. [CrossRef]

6. Yang, D.-K. Fundamentals of Liquid Crystal Devices; John Wiley \& Sons: Hoboken, NJ, USA, 2014.

7. Lucchetti, L.; Kushnir, K.; Zaltron, A.; Simoni, F. Light controlled phase shifter for optofluidics. Opt. Lett. 2016, 41, $333-335$. [CrossRef]

8. Lucchetti, L.; Kushnir, L.K.; Reshetnyak, V.Y.; Ciciulla, F.; Zaltron, A.; Sada, C.; Simoni, F. Light-induced electric field generated by photovoltaic substrates investigated through liquid crystal reorientation. Opt. Mater. 2017, 73, 64-69. [CrossRef]

9. Habibpourmoghadam, A.; Lucchetti, L.; Evans, D.R.; Reshetnyak, V.Y.; Omairat, F.; Shafforz, S.L.; Lorenz, A. Laser-induced erasable patterns in a $\mathrm{N}^{*}$ liquid crystal on an iron doped lithium niobate surface. Opt. Express 2017, 25, 26148-26159. [CrossRef] [PubMed]

10. Volk, T.; Wöhlecke, M. Lithium Niobate: Defects, Photorefraction and Ferroelectric Switching; Springer Science \& Business Media: Cham, Switzerland, 2008.

11. Fridkin, V.M. Bulk photovoltaic effect in non-centrosymmetric crystals. Crystallogr. Rep. 2001, 46, 654-658. [CrossRef]

12. Lucchetti, L.; Kushnir, K.; Zaltron, A.; Simoni, F. Liquid crystal cells based on photovoltaic substrates. J. Eur. Opt. Soc. 2016, 11, 16007. [CrossRef]

13. Kong, Y.; Liu, S.; Xu, J. Recent advances in photorefraction of doped lithium niobate crystals. Materials 2012, 5, 1954. [CrossRef]

14. Vittadello, L.; Zaltron, A.; Argiolas, N.; Bazzan, M.; Rossetto, N.; Signorini, R.J. Photorefractive direct laser writing. Phys. D Appl. Phys. 2016, 49, 125103. [CrossRef]

15. Günter, P.; Huignard, J.P. Photorefractive Materials and Their Applications 2: Materials; Springer: Berlin/Heidelberg, Germany, 2007.

16. Bonfadini, S.; Ciciulla, F.; Criante, L.; Zaltron, A.; Simoni, F.; Reshetnyak, V.; Lucchetti, L. Optofluidic platform using liquid crystals in lithium niobate microchannel. Sci. Rep. 2019, 9, 1062. [CrossRef] [PubMed]

17. Yamaguchi, R. Thresholdless Electro-Optical Property in Quasi Homogeneous and Homeotropic Liquid Crystal Cells Using Weak Anchoring Surfaces. IEICE Trans. Electron. 2019, E102-C, 810-812. [CrossRef]

18. Barbero, G.; Barberi, R. Critical thickness of a hybrid aligned nematic liquid crystal cell. J. Physique 1983, 44, 609-616. [CrossRef]

19. Barbero, G.; Simoni, F.; Aiello, P. Nonlinear optical reorientation in hybrid aligned nematics. J. Appl. Phys. 1984, 55, 304-311. [CrossRef]

20. Schafforz, S.L.; Nordendorf, G.; Nava, G.; Lucchetti, L.; Lorenz, A. Formation of relocatable umbilical defects in a liquid crystal with positive dielectric anisotropy induced via photovoltaic fields. J. Mol. Liq. 2020, 307, 112963. [CrossRef]

21. Ciampolillo, M.V.; Zaltron, A.; Bazzan, M.; Argiolas, N.; Sada, C. Quantification of iron (Fe) in lithium niobate by optical absorption. Appl. Spectrosc. 2011, 65, 216-220. [CrossRef]

22. Bettella, G.; Pozza, G.; Kroesen, S.; Zamboni, R.; Baggio, E.; Montevecchi, C.; Zaltron, A.; Gauthier-Manuel, L.; Mistura, G.; Furlan, C.; et al. Lithium niobate micromachining for the fabrication of microfludici droplet generators. Micromachines 2017, 8, $1954-1971$. [CrossRef]

23. Simoni, F. Nonlinear Optical Properties of Liquid Crystals and Polymer Dispersed Liquid; Word Scientific: Singapore, 1997.

24. Buse, K.; Imbrock, J.; Kratzig, E.; Peithmann, K. Photorefractive Effects in $\mathrm{LiNbO}_{3}$ and $\mathrm{LiTaO}_{3}$. In Photorefractive Materials and Their Applications 2; Gunter, P., Huignard, J.P., Eds.; Springer: Berlin/Heidelberg, Germany, 2007.

25. Carrascosa, M.; Agullo-Lopez, F. Theoretical modeling of the fixing and developing of holographic gratings in $\mathrm{LiNbO}_{3} . \mathrm{J}_{\text {. Opt. }}$ Soc. B 1990, 7, 2317. [CrossRef]

26. Kukhtarev, N.V.; Markov, V.B.; Odulov, S.G.; Soskin, M.S.; Vinetskii, V.L. Holographic storage in electrooptic crystals. I. steady state. Ferroelectrics 1979, 22, 949. [CrossRef]

27. Valley, G.C.; Lam, J.F. Theory of photorefractive effects in electro-optic crystals. In Photorefractive Materials and Their Applications 1; Gunter, P., Huignard, J.P., Eds.; Springer: Berlin/Heidelberg, Germany, 2007.

28. Yeh, P.; Gu, C. Optics of Liquid Crystal Displays, 2nd ed.; Wiley: Hoboken, NJ, USA, 2010. 